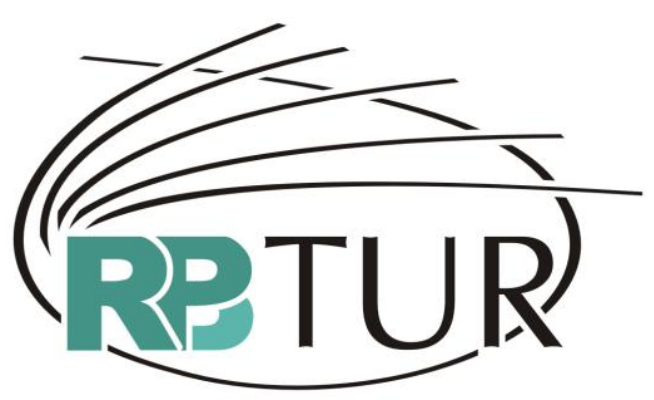

REVISTA BRASILEIRA DE PESQUISA EM TURISMO

\title{
TURISMO Y RECURSOS TERMALES: EL CASO DE LA INSTALACIÓN DEL PARQUE TERMAL DE LA CIUDAD DE VICTORIA, ENTRE RÍOS, ARGENTINA
}

\author{
TURISMO E RECURSOS TERMAIS: O CASO DA INSTALAÇÃO \\ DO PARQUE TERMAL DA CIDADE DE VICTORIA, ENTRE RIOS, \\ ARGENTINA
}

\begin{abstract}
TOURISM AND THERMAL RESOURCES: THE CASE OF THE THERMAL PARK INSTALLMENT AT VICTORIA CITY, ENTRE RIOS, ARGENTINA
\end{abstract}

Julia Piñeiro Carreras ${ }^{1}$

\begin{abstract}
Resumen: En este artículo analizo el proceso de instalación de un parque termal (2003-2010) que se da en el contexto de la transformación de la localidad de Victoria (Entre Ríos, Argentina), en una ciudad turística. Para ello, utilizo materiales producidos en una investigación antropológica de carácter etnográfico, así como una diversidad de documentos periodísticos y de carácter legislativo. Con el fin de mostrar el carácter activo de específicos actores locales en la turistificación de la ciudad, reconstruyo el proceso de instalación del parque termal contextualizándolo en el marco de las discusiones tendientes a la modificación de las normativas referidas al aprovechamiento turístico del agua termal en la provincia. Atiendo especialmente al papel que, en la llegada de este nuevo emprendimiento, ocuparon específicos actores sociales del ámbito empresarial y estatal, y a las relaciones que entablaron entre sí.
\end{abstract}

Palabras clave: Turismo. Parque termal. Empresarios. Estado. Etnografía. Victoria (Entre Ríos, Argentina).

Resumo: O artigo analisa o processo de instalação de um parque termal (2003-2010) no contexto da transformação da localidade de Victoria (Entre Ríos, Argentina), em uma cidade turística. Para isso, utilizo tanto materiais produzidos a partir de uma

1 Licenciada en Ciencias Antropológicas Orientación Sociocultural (Facultad de Filosofía y Letras, UBA), Magister en Antropología Social (IDES-IDAES, Universidad de San Martín), Doctoranda en Antropología (Facultad de Filosofía y Letras, UBA), Becaria doctoral de CONICET (con lugar de trabajo en el Instituto de Ciencias Antropológicas, FFyL, UBA), Docente en la Facultad de Ciencias Sociales, UBA. Email: juliapini@gmail.com 
PIÑEIRO CARRERAS, Julia. Turismo y recursos termales: el caso de la instalación del parque termal de la ciudad de Victoria, Entre Ríos, Argentina. Revista Brasileira de Pesquisa em Turismo. v.5, n.3, p.383405, dez.2011.

ISSN: $1982-6125$

pesquisa antropológica de caráter etnográfico, quanto uma diversidade de documentos jornalísticos e governamentais. Com o intuito de mostrar o caráter ativo de atores locais específicos na turistificação da cidade, reconstruo o processo de instalação do parque termal, explorando sua relação com os debates sobre a modificação das normativas referidas ao aproveitamento das águas termais na província. Considero com especial atenção o papel que, na chegada do novo empreendimento, ocuparam atores sociais específicos do âmbito empresário e estatal, e as relações que estabeleceram entre sim.

Palavras chave: Turismo. Parque termal. Empresários. Estado. Etnografia. Victoria (Entre Ríos, Argentina).

Abstract: The article analyzes the installment process of a thermal park (2003-2010) along with the transformation of Victoria City (Entre Ríos, Argentina) into a touristic place. Data issued from an ethnographic research are used, as well as several journalistic and legal documents. The process leading to the installment of the thermal park is reconstructed to point out the active role of some local actors in the tourismification of the city, while discussions about changing the rules on thermal water use for touristic profit were held. The focus is on the role of specific social actors in privet and public sectors and in the way they interacted during the process.

Keywords: Tourism. Thermal park. Entrepreneurs. State. Ethnography. Victoria (Entre Ríos, Argentina).

\section{Introducción}

La antropología del turismo ha estado, desde sus inicios en la década del '60, signada por la búsqueda de comprender las transformaciones que éste genera en las llamadas 'sociedades receptoras' o 'de destino'. Por mucho tiempo, los antropólogos recurrieron al concepto de 'impacto', o a alguno de sus parientes semánticos, para describir y explicar aquello que el turismo estaba generando en las localidades que lo recibían. En las últimas décadas, la apelación explícita a la noción de 'impacto' ha sido progresivamente abandonada, en tanto se ha considerado que la misma conlleva ciertos supuestos problemáticos, como ser el de una cierta homogeneidad de las sociedades receptoras, y el del turismo como un agente externo 'golpeando' ('impactando en') esas sociedades (Barretto, 2007).

Sin embargo, hay quienes sostienen que estamos lejos de haber dejado de pensar en esos términos aún cuando nuestro lenguaje se haya modificado (Nogués Pedregal, 2009). Los procesos de turistificación se nos presentan 
PIÑEIRO CARRERAS, Julia. Turismo y recursos termales: el caso de la instalación del parque termal de la ciudad de Victoria, Entre Ríos, Argentina. Revista Brasileira de Pesquisa em Turismo. v.5, n.3, p.383405, dez.2011.

ISSN: $1982-6125$

como una oportunidad privilegiada para ver los roles activos que toman específicos actores locales, y que no pueden ser deslindados de las relaciones que éstos entablan entre sí y con otros actores identificados con planos supralocales. Es desde esta idea que en el presente artículo analizo el proceso de instalación de un parque termal que se dio en el marco de la turistificación de la ciudad de Victoria (Entre Ríos, Argentina). Dicho emprendimiento fue impulsado por un grupo empresario y su desarrollo tuvo lugar entre 2003 y 2010, año en el que fue abierto al público.

$\mathrm{Si}$, tal como sostiene Bianchi, los diferentes actores involucrados en el desarrollo turístico de localidades particulares están "dotados de desiguales capacidades de explotar las oportunidades económicas que se les presentan, dependiendo de su capacidad para concebir, apropiarse de, regular y controlar los medios de la producción turística"” (2003, p.18), el caso que nos ocupa es especialmente interesante ya que los parques termales se basan en el aprovechamiento de un recurso que, en función de la legislación argentina, se considera bajo dominio de las provincias. A esto se suma el hecho de que, en el período de creación del parque de Victoria, el marco regulatorio del recurso termal estuviera siendo justamente objeto de importantes modificaciones. Dentro de este contexto de redefinición de normativas me interesa centrarme en la participación que tuvieron empresarios, funcionarios municipales, legisladores provinciales, autoridades de organismos provinciales y nacionales, concejales, etc., y cuáles fueron las relaciones que en el contexto del proceso de instalación del parque fueron construyendo.

Los materiales en los que se basa este trabajo fueron producidos en el marco de una investigación etnográfica desarrollada entre los años 2007 y 2009, y que incluyó - además del uso de las técnicas de observación participante y entrevistas abiertas- un trabajo de relevamiento de fuentes periodísticas locales. Asimismo, apelo al análisis de otras fuentes documentales como leyes, ordenanzas, versiones taquigráficas de sesiones legislativas, decretos etc. .

\footnotetext{
${ }^{2}$ Traducción propia.
} 
PIÑEIRO CARRERAS, Julia. Turismo y recursos termales: el caso de la instalación del parque termal de la ciudad de Victoria, Entre Ríos, Argentina. Revista Brasileira de Pesquisa em Turismo. v.5, n.3, p.383405, dez.2011.

ISSN: $1982-6125$

En el primer apartado, me refiero brevemente a las características principales de la turistificación de Victoria a fin de contextualizar el proceso de creación del parque termal. En el segundo apartado, me dedico a reconstruir los inicios del proyecto enmarcándolo en la reformulación de las normativas provinciales referidas al aprovechamiento turístico del recurso termal, y atendiendo especialmente al rol que asumieron los empresarios inversores así como una diversidad de políticos y funcionarios del ámbito local y supralocal. Manteniendo esa atención, en el tercer apartado me concentro en la formulación de la denominada "Ley de Termas". En las conclusiones, enfatizo la necesidad de prestar especial atención a las relaciones que, en los procesos de turistificación, entablan diferentes actores que se conciben a sí mismos como formando parte de ámbitos distintos: el de lo privado y el de lo estatal.

\section{Una ciudad en transformación}

A la vera del río Paraná se encuentra Victoria, ciudad cabecera del departamento homónimo ${ }^{3}$, ubicado al sudoeste de la provincia de Entre Ríos. Hasta hace algunos años, la ciudad era percibida por sus propios habitantes como dedicada casi exclusivamente, por un lado, a las actividades terciarias derivadas de las agropecuarias - desarrolladas en la zona de campaña del Departamento $-{ }^{4}, y$, por el otro, a la pesca que se lleva adelante en la zona del valle fluvial ${ }^{5}$.

Hoy, Victoria es localmente considerada como una ciudad que, amén de seguir dependiendo en gran medida de esas actividades productivas primarias, se está transformando en una ciudad turística ${ }^{6}$. La actividad turística no es nueva en la ciudad y puede rastrearse, por lo menos, hasta la década del setenta. Sin embargo, tuvo un crecimiento notable cuando en 2003 una mega

3 La provincia de Entre Ríos se encuentra dividida en 17 Departamentos. El Departamento Victoria está conformado, además de por su cabecera, por nueve distritos.

${ }^{4}$ En la actualidad, cultivo de soja, trigo y maíz, y ganadería de invernada.

${ }^{5} \mathrm{En}$ las islas que forman este valle fluvial también se llevan a cabo actividades de engorde de ganado bovino, caza comercial (nutria), apicultura, y, de manera incipiente, agricultura.

${ }^{6}$ Uso la cursiva para referir expresiones nativas. 
PIÑEIRO CARRERAS, Julia. Turismo y recursos termales: el caso de la instalación del parque termal de la ciudad de Victoria, Entre Ríos, Argentina. Revista Brasileira de Pesquisa em Turismo. v.5, n.3, p.383405, dez.2011.

ISSN: $1982-6125$

obra vial la conectó con la ciudad de Rosario, de la vecina provincia de Santa Fe. El Puente Nuestra Señora del Rosario es, a ciencia cierta, un conjunto de puentes y terraplenes de 59,4 km que atraviesa el valle fluvial del Paraná y es localmente designado como el puente Victoria-Rosario o, simplemente, el puente. El mismo unió a dos ciudades de escalas notablemente diferentes: mientras que hacia 2003 Rosario contaba con una población de más de 900.000 habitantes, Victoria tenía menos de 30.0007.

Las posibilidades turísticas que la unión podría generar fue uno de los temas que tomó relevancia pública a partir del momento en que el puente comenzó a construirse, en $1998^{8}$. En efecto, en los años que siguieron al comienzo de las obras, y aún con más fuerza una vez que el puente estuvo habilitado, los emprendimientos vinculados al sector turístico de capitales fundamentalmente locales comenzaron a reproducirse en la ciudad. Entre ellos se destacan, tanto por sus dimensiones como por las expectativas que movilizaron, un hotel-casino inaugurado en $2005^{9}$ y el parque acuático termal a cuyo proceso de instalación se dedica el presente trabajo.

\section{Un emprendimiento, un recurso natural}

La cuestión del papel que, en la instalación del parque termal ubicado en las afueras de la ciudad de Victoria, cabía al grupo empresario impulsor del proyecto y a diferentes instituciones estatales de nivel local y provincial, fue uno de los puntos que surgieron en la entrevista que, en febrero de 2008, realicé al vocero de los inversores. Por ese entonces, las obras del parque ya habían comenzado y el pozo del cual se extraería el agua estaba listo. Según expresó el empresario, habían sido ellos, los miembros del grupo que él lideraba, quienes habían hecho una "inversión de riesgo" al hacer los estudios

7 Según el Censo Nacional de Población, Hogares y Viviendas de 2001, el municipio de Rosario tenía 909.397 y el de Victoria 28.492

8 Para un análisis del proceso político que derivó en la construcción de la obra vial véase Gaztañaga, 2010.

9 Para un análisis del proceso de instalación del hotel-casino véase Piñeiro Carreras 2009 y 2011. 
PIÑEIRO CARRERAS, Julia. Turismo y recursos termales: el caso de la instalación del parque termal de la ciudad de Victoria, Entre Ríos, Argentina. Revista Brasileira de Pesquisa em Turismo. v.5, n.3, p.383405, dez.2011.

ISSN: $1982-6125$

en busca del agua termal, la perforación de casi 1.000 metros de profundidad para alcanzarla y, luego, todas las tareas de movimiento de tierra y edificaciones necesarias. Todavía faltaban obras para terminar el emprendimiento: en sus palabras, "un megaproyecto" que incluía, más allá del parque acuático propiamente dicho - que contendría, además de una diversidad de piletas y juegos acuáticos, un "museo del agua", un spa, y varios restaurantes - la construcción, en una segunda etapa, de cinco hoteles de entre cuatro y cinco estrellas.

Convencidos de que "una comunidad se hace con el esfuerzo de todos", ellos hacían su parte. Pero esperaban que "el Estado" hiciera la suya. Se necesitaban obras de infraestructura (cloacal, vial etc.) que correspondían de alguna u otra manera a lo que el empresario llamaba "Estado" o "sector público" y que, según el contexto, significaba "el municipio", "la provincia", o algunas de sus instituciones o funcionarios. Una cuestión que por entonces preocupaba especialmente al empresario, era que el terreno sobre el que se apostaba el parque no formaba parte del ejido urbano. Eso implicaba que ciertas obras y servicios que se esperaba proveyera el municipio, aún no pudieran garantizarse ${ }^{10}$. Respecto de esta cuestión en particular (que, como veremos, acabaría resolviéndose) el empresario me dijo:

Está en la legislatura el proyecto de ley que hace la ampliación del ejido. Nosotros lo solicitamos. Nosotros queremos que el municipio se involucre. Por suerte el municipio, o por lo menos el presidente municipal, está muy consustanciado con este emprendimiento y quiere poner a disposición todo su esfuerzo. Pero lamentablemente con el esfuerzo del presidente municipal no alcanza. Esperemos que todos comprendan, todos los que tienen que intervenir comprendan que hay que acompañar al sector privado. Esa es la cuestión.

La reconstrucción del proceso de instalación del parque, que había comenzado varios años antes del comienzo de mi investigación, me permitió

${ }^{10}$ De acuerdo con la estructura institucional de Entre Ríos, las intendencias de las ciudades cabeceras de Departamento (como lo es Victoria), ejercen una jurisdicción que se limita a la de su ejido o casco urbano, dependiendo el resto del territorio departamental directamente de la Gobernación, con la sola mediación de Juntas de Gobierno locales. 
PIÑEIRO CARRERAS, Julia. Turismo y recursos termales: el caso de la instalación del parque termal de la ciudad de Victoria, Entre Ríos, Argentina. Revista Brasileira de Pesquisa em Turismo. v.5, n.3, p.383405, dez.2011.

ISSN: $1982-6125$

entender a qué se refería el empresario al decir que el intendente, que cumplía su segundo mandato por el justicialismo ${ }^{11}$, estaba "consustanciado" $\mathrm{y}$, también, cuáles habían sido, y eran, los otros actores de lo que aquel llamaba "sector público" que habían estado involucrados en la consecución del proyecto.

La idea de hacer un parque termal había surgido de la Sociedad Anónima Sol de Victoria, grupo empresario conformado por personas provenientes de Victoria y de Rosario ${ }^{12}$ que, al momento de encarar este nuevo proyecto, ya se encontraba embarcado en el de montar el primer hotel-casino de la ciudad ${ }^{13}$. A principios de 2003 los inversores encargaron un estudio de "Prospección geológica para la detección de acuíferos profundos" y, unos meses más tarde, se conoció que habían adquirido, una parte en propiedad y otra en arriendo, unas 40 hectáreas ubicadas a $8 \mathrm{~km}$ del centro de la ciudad frente a la llamada Laguna del Pescado.

Si el emprendimiento se concretaba, Victoria entraría en el mapa termal de Entre Ríos. El turismo termal se había iniciado en la provincia a mediados de la década del '90, habiendo sido Federación la localidad pionera (Catullo, 2005; Wallingre, 2008; Ramírez, 2011). Desde fines de esa década y la primera mitad de la siguiente, se estaba registrando un crecimiento notable de la actividad abriéndose centros termales en distintas localidades como Villa Elisa, Concordia, Chajarí, Colón, La Paz, San José, María Grande y Gualeguaychú (Wallingre, 2008). El marco regulatorio referido al

${ }^{11}$ En referencia al Partido Justicialista, fundado por Juan Domingo Perón. En los años de que se ocupa este artículo, existieron grandes divergencias al interior del peronismo tanto en la localidad de Victoria como a nivel provincial y nacional. Por razones de espacio no abundaré aquí en esta cuestión. El intendente ocupó tres mandatos: 1991-1995 y 2003-2007 por el Partido Justicialista, y 2007-2011 por el Frente Justicialista para la Victoria.

12 Más adelante se creó una nueva sociedad anónima bajo el nombre Victoria del Agua con parte de los empresarios que conformaban Sol de Victoria S.A., entre los cuales estaba el vocero del grupo. En la entrevista que le hice en 2008, aunque sin hacer referencia explícita a los vaivenes societarios, enfatizó el carácter local del grupo al hacer referencia a los motivos que lo habían llevado a generar el proyecto termal: "[lo hacemos] porque somos todos victorienses. (...)somos hijos de esta tierra, hemos vivido en esta tierra, y nuestros beneficios los queremos volcar acá".

${ }^{13}$ Si bien se trata de un hotel-casino, el alojamiento es propiedad de esta sociedad anónima, mientras que el casino propiamente dicho pertenece a otra empresa propietaria de otros establecimientos de juegos de azar en el país. 
PIÑEIRO CARRERAS, Julia. Turismo y recursos termales: el caso de la instalación del parque termal de la ciudad de Victoria, Entre Ríos, Argentina. Revista Brasileira de Pesquisa em Turismo. v.5, n.3, p.383405, dez.2011.

ISSN: $1982-6125$

aprovechamiento de las aguas termales, que había ido sufriendo modificaciones desde el primer antecedente, de 1993, se encontraba, al iniciarse el proyecto en Victoria, en pleno debate en virtud de la notable expansión que vivía la actividad. En 2003, la norma vigente era la creada por el decreto no 3413 de agosto de 1998, pero ya se daban en la provincia las discusiones referidas a la necesidad de que existiera una Ley de Termas. En efecto, todo el proceso de proyección y concreción del emprendimiento en Victoria se dio simultáneamente a la reformulación del marco normativo referido al aprovechamiento turístico de las aguas termales.

Un primer elemento a tener en consideración para comprender el proceso de instalación del parque termal es el hecho de que, en virtud del artículo 124 de la Constitución Nacional argentina, son las provincias quienes tienen el dominio sobre "los recursos naturales existentes en su territorio". Es decir que los promotores del emprendimiento debían obtener un permiso de la Gobernación para "explorar" y "perforar" el suelo en busca del agua termal. En este sentido, el estado provincial tendría un rol fundamental, en tanto su aprobación se constituía en una condición de posibilidad para la creación del parque. Pero también el estado municipal ocuparía un papel importante aún cuando la zona en la que se apostaría el emprendimiento se encontraba fuera de los límites del ejido urbano $y$, por tanto, más allá de su control administrativo. De hecho, el intendente del municipio de Victoria participó de las gestiones que los empresarios debieron llevar a cabo frente a las autoridades competentes del gobierno provincial a la hora de obtener, primero, el permiso de exploración y, luego, otra serie de habilitaciones necesarias. De allí, que el empresario expresara que el Intendente había estado "consustanciado" con el proyecto.

Según lo publicó el semanario local Paralelo 32, el proyecto fue presentado formalmente por el grupo inversor ante el Subsecretario de Turismo provincial, en mayo de 2004, en una reunión en la que estuvieron acompañados por autoridades municipales. Luego de aquel encuentro, al ser entrevistado por el semanario, el Intendente expresó que se emprendía un 
camino que "[llevaría] su tiempo" y que el trámite no iba a ser "nada corto". Además, explicitando cuáles eran, y serían, los protagonistas de ese largo proceso, dijo: "el compromiso y la predisposición de los empresarios, de la Municipalidad y de la provincia, existen y son los mejores". La Municipalidad agregó - sería "socia" del proyecto, "a través de una contraprestación en cuanto a la construcción de accesos e infraestructura" (Paralelo 32, 22/5/2004). Y aquí vale la pena llamar la atención a cómo el presidente municipal estaba definiendo esa "sociedad". Tal como lo expresaba, el rol que en la misma le cabría al gobierno local sería el de "contraprestar" proveyendo de cierta infraestructura que el emprendimiento, privado, necesitaría. En el caso del parque termal de Victoria, había sido el grupo empresario el encargado de solventar y realizar la prospección, y también debería ocuparse de la perforación, en el caso de obtener el permiso que solicitaba al gobierno provincial. Esto no es un dato menor si tenemos en cuenta que en otras de las ciudades entrerrianas con termas, fue el municipio quien se hizo cargo de las distintas etapas de instalación, incluyendo la de la siempre onerosa tarea de perforación. ${ }^{14}$

La noticia de la entrega del proyecto del parque a autoridades provinciales no fue la primera referencia que el semanario Paralelo 32 hacía al tema de la explotación termal en la provincia. En efecto, ya venía instalando el tema como 'de interés' desde el momento de conocerse, en 2003, la idea de "los dueños del hotel" (como suele denominarse al grupo empresario), de invertir en un parque termal. Desde ese momento, las notas referidas no sólo al proyecto local sino a las termas entrerrianas en general se multiplicaron. La "cuestión termal" estaba en el candelero a nivel provincial, pues por entonces una importante cantidad de localidades emprendían proyectos que implicaban el aprovechamiento del recurso termal $y$, por tanto, solicitaban permisos de

14 Según lo expone Ramírez (2011) para el caso de Federación, en esa localidad, fue el gobierno municipal el que impulsó y ejecutó la totalidad de las obras (incluyendo la prospección, perforación y construcción del parque termal). Hasta la actualidad el parque es de propiedad y gestión municipal. En el caso de San José, el parque termal existente en la localidad fue promovido desde el municipio y estuvo bajo su egida desde su proyección en 2003 hasta 2007, cuando fue concesionado (Wallingre, 2011). 
PIÑEIRO CARRERAS, Julia. Turismo y recursos termales: el caso de la instalación del parque termal de la ciudad de Victoria, Entre Ríos, Argentina. Revista Brasileira de Pesquisa em Turismo. v.5, n.3, p.383405, dez.2011.

ISSN: $1982-6125$

exploración. En enero de 2004 el semanario publicó unas líneas bajo el título "El gruyere termal" donde informaba que el gobierno entrerriano había suspendido momentáneamente los permisos para hacer estudios o realizar perforaciones con el fin de explotar los recursos termales. La suspensión había sido dispuesta por un Dictamen del Consejo Asesor para asuntos termales ${ }^{15}$ del 30 de diciembre de 2003, por el cual se decidía no estudiar ningún expediente generado con posterioridad a esa fecha por el término de un año.

¿Qué sucedería en este contexto con el parque termal que se proyectaba en Victoria? Esa fue una de las consultas que un periodista de Paralelo 32 hizo al vocero de los inversores. Como respuesta, él "reconoció" que otros emprendimientos termales habían sido paralizados en distintos departamentos de la provincia por decisión del gobierno, pero aclaró que el único que mantenía "vigencia" era el que correspondía a Victoria, aunque sin explicar los motivos para ello (Paralelo 32, 24/04/2004) ${ }^{16}$. En la misma edición que luego, en mayo, informaría sobre la entrega oficial del proyecto al Subsecretario de Turismo, se publicaba otra nota bajo el título "Replantean la explotación del recurso", que relataba otra reunión referida a un Plan de Reordenamiento del Recurso Termal en la que también había participado dicho funcionario, junto con el director General de Minería de Entre Ríos y el Jefe del Departamento de Geometría del Instituto de Geología y Recursos Minerales de la Secretaría de Minería de la Nación. El objetivo era, según habría dicho el Subsecretario de Turismo, hacer un replanteo de los recursos y de la explotación de acuerdo al impacto ambiental, así como verificar el potencial del recurso geotérmico de la provincia para saber en qué áreas se justificaba una perforación para, así, poder orientar a los inversionistas 0 intendentes que quisieran realizar emprendimientos termales (Paralelo 32, 22/5/2004).

${ }^{15}$ Era ese Consejo la Autoridad de Aplicación designada por el decreto 3413/98 que, tal como señalamos, era la norma vigente en ese momento.

${ }^{16}$ Si bien el periódico local Paralelo 32 refirió que la presentación del proyecto "a la provincia" fue en mayo de 2004, no dispongo de información respecto de si se había hecho otra presentación con anterioridad a la fecha en que se dispuso la suspensión de recepción de expedientes el 30 de diciembre de 2003. 
Coadyuvando a la relevancia local del tema y a los giros dramáticos que el mismo parecía adquirir, Paralelo 32 siguió ocupándose de la cuestión. Uno de sus redactores hizo referencia en agosto del mismo año, a versiones sobre el peligro de contaminación del Acuífero Guaraní (del cual provendría el agua termal), "justo en este momento, cuando capitales locales proyectan montar un parque temático con termas" (Paralelo 32, 24/8/2004). En septiembre, el periódico publicó una nota bajo el título "El termalismo entrerriano en la encrucijada", en la que se referían datos sobre la cantidad de permisos otorgados antes de la suspensión y la existencia de sectores ambientalistas en la provincia que mostraban preocupación y denunciaban que muchos emprendimientos se habían aprobado sin cumplir con las especificaciones técnicas exigidas (Paralelo 32, 25/9/2004). En octubre, se publicó una nota titulada "Aguas Termales y Hoteles. Se caen y se levantan propuestas todas las semanas", donde se mencionaba que el Intendente de Victoria se había reunido con el presidente de la Comisión de Aguas Termales de la provincia para conocer el estado del proyecto que involucraba a la ciudad. En espera de la evaluación de la Dirección de Hidráulica de la Provincia, el presidente municipal había dicho que ese era "el último estamento que [tendría] que recorrer el expediente" y que esperaban "tener la factibilidad para el término de noviembre". Entretanto, informaba el semanario, los inversores fueron recibidos por el Secretario de Turismo de la Nación, ante quien expusieron las "virtudes y proyectos" que aspiraban concretar con la obra (Paralelo 32, 23/10/2004).

El 23 de diciembre de 2004 un decreto del ejecutivo provincial, reafirmando un dictamen del Consejo Asesor, dispuso no recibir nuevas "presentaciones" hasta tanto se promulgara la "Ley de Marco Regulatorio y Creación del Ente Regulador Provincial de Termas de la Provincia de Entre Ríos", de la cual -tal como se exponía en los considerandos del decreto- había un proyecto presentado por cinco Legisladores del Bloque del Partido Justicialista en trámite legislativo en la Cámara de Diputados (Decreto $\mathrm{n}^{\circ}$ 
PIÑEIRO CARRERAS, Julia. Turismo y recursos termales: el caso de la instalación del parque termal de la ciudad de Victoria, Entre Ríos, Argentina. Revista Brasileira de Pesquisa em Turismo. v.5, n.3, p.383405, dez.2011.

ISSN: $1982-6125$

7137/04) ${ }^{17}$. En el decreto, además, el ejecutivo daba el visto bueno a la decisión de suspender las exploraciones, en tanto consideraba que el Consejo que había sido creado como Autoridad de Aplicación por el Decreto n 3413/98 no había ejercido "su función debidamente desde el año 2000 hasta fines de 2003". Esa situación había generado, "un cúmulo de solicitudes de perforación, sin que existiera una política de desarrollo ni criterio de otorgamiento de permisos", los cuales se habían dado "en muchos casos sin cumplimentar los requerimientos establecidos" por la normativa vigente, generando por ello incertidumbre sobre los efectos posteriores en materia de impacto ambiental (Decreto $\left.\mathrm{n}^{\circ} 7137 / 04\right)$.

En ese contexto de creación de nuevas normativas, cuando el asunto estaba aún bajo la órbita del decreto no 3.413/98, Sol de Victoria S.A. obtuvo su Certificado de Prefactibilidad de Exploración y Perforación del Recurso Termal el 5 de abril de 2005 gracias al decreto $n^{\circ}$ 1.355/05 firmado por el entonces Gobernador, Jorge Pedro Busti ${ }^{18}$ y su Ministro de Gobierno, Educación y Justicia Sergio Urribarri, quien sería luego su sucesor en la gobernación ${ }^{19}$. Ese mismo día por la tarde, las autoridades municipales convocaron a una conferencia de prensa en el despacho municipal para anunciar la buena nueva junto al vocero de los inversores y a un diputado provincial victoriense ${ }^{20}$.

\section{La ley de termas: ordenar la explotación}

La Ley de Termas fue sancionada unos meses después, el 20 de diciembre de 2005, por la Cámara de Diputados provincial y llevaba por objeto, según su primer artículo, establecer el marco regulatorio del manejo de los

17 El proyecto de ley mencionado en el decreto había sido presentado ante la Cámara de Diputados el 17 de noviembre de 2004 y pasado a la comisión de Tierra y Obras Publicas, Recursos Naturales y Medio Ambiente.

${ }_{18}$ Por el justicialismo en los períodos 1987-1991; 1995-1999; 2003-2007.

${ }^{19}$ Frente Justicialista para la Victoria 2007-2011.

20 Perteneciente al Bloque del Partido Justicialista. Fue diputado provincial en los períodos 2003-2007 y 2007-2011. Los diputados son elegidos por el pueblo de la provincia, en distrito único. 
PIÑEIRO CARRERAS, Julia. Turismo y recursos termales: el caso de la instalación del parque termal de la ciudad de Victoria, Entre Ríos, Argentina. Revista Brasileira de Pesquisa em Turismo. v.5, n.3, p.383405, dez.2011.

ISSN: $1982-6125$

recursos termales que se gestionaran con fines terapéuticos, medicinales, recreativos y/o turísticos, cuyo lugar de alumbramiento se situara dentro de la jurisdicción provincial; definir los lineamientos de política general en esta materia; y crear un órgano específico que sería su Autoridad de Aplicación. Su alcance incluía tanto el estudio de los recursos termales, como la planificación de su uso, exploración y explotación; la determinación del tratamiento y disposición de los recursos residuales del aprovechamiento; y la ordenación, fomento y promoción de la actividad termal en la provincia. El "órgano específico" que se creaba era el Ente Regulador de Recursos Termales de Entre Ríos (E.R.R.T.E.R).

Pero el Poder Ejecutivo vetó la ley por completo el 23 de enero de 2006 (Decreto $\mathrm{n}^{\circ}$ 37/2006). En los considerandos del veto se cuestionaba el hecho de que, al crearse el E.R.R.T.E.R, el manejo del recurso hídrico quedaría dividido en más de un organismo: este ente se encargaría específicamente de las aguas termales, mientras que las aguas no subterráneas quedarían bajo la egida del Consejo Regulador de Uso de Fuentes de Agua (C.O.R.U.F.A), la Dirección de Hidráulica, y la Dirección de Obras Sanitarias. El problema, sostenía el Poder Ejecutivo, residía en que, de ese modo, se iba en contra de compromisos firmados por la provincia con "la Nación". Se trataba del Acuerdo Federal del Agua, que databa del año 2003, en el que se estipulaba que el agua en todas sus formas debía ser tratada como un recurso único y ser controlada, por tanto, por una sola autoridad por provincia. Por otro lado, los considerandos del veto hacían énfasis en la cuestión "ambiental" (especialmente sensible en la provincia en aquel momento por un conflicto generado por la instalación de pasteras en la margen uruguaya del Río Uruguay $^{21}$ ). En ese sentido, se remarcaba la necesidad de garantizar el cumplimiento de las "Normas Técnicas de construcción de Pozos para la

21 El conflicto se había iniciado en 2003 ante los proyectos de instalación de fábricas de celulosa de capitales finlandeses y españoles en la localidad de Fray Bentos, en la República Oriental del Uruguay, separada por el Río Uruguay de la localidad entrerriana de Gualeguaychú, donde se dio un importante movimiento ambientalista de oposición a las pasteras. 
PIÑEIRO CARRERAS, Julia. Turismo y recursos termales: el caso de la instalación del parque termal de la ciudad de Victoria, Entre Ríos, Argentina. Revista Brasileira de Pesquisa em Turismo. v.5, n.3, p.383405, dez.2011.

ISSN: $1982-6125$

explotación de Aguas Subterráneas", que habían sido confeccionadas por el presidente del denominado Proyecto Piloto Concordia-Salto en el marco del Proyecto para la Protección Ambiental y Desarrollo Sustentable del Acuífero

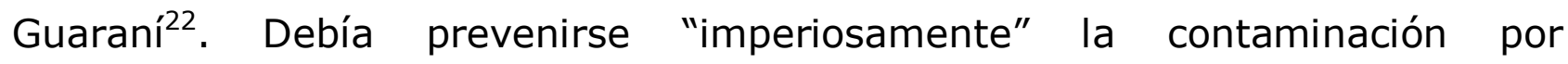
salinización, para lo cual era necesario explicitar en la ley la "obligatoriedad" de realizar pozos de "reinyección".

Al ocuparse de la cuestión del veto, el semanario Paralelo 32 publicó información sobre un viaje que el Intendente había hecho esa misma semana a la capital provincial. Consultado por el periódico, el presidente municipal aclaraba que la decisión del ejecutivo provincial no afectaba de ninguna forma el proyecto que se había aprobado en Victoria. La pregunta provenía del hecho de que, si bien para ese entonces la empresa gozaba del permiso para "explorar", las obras recién estaban por comenzar. El siguiente número del semanario volvió a ocuparse del tema reproduciendo las palabras que el Subsecretario de Turismo de la provincia había vertido en una entrevista radial. El funcionario había dicho que el veto lo "había tomado por sorpresa" (Paralelo 32, 4/2/2006).

Una vez producido el veto, la Asamblea Legislativa entrerriana (conformada por las dos Cámaras) podía aceptarlo o rechazarlo. El asunto se trató el 23 de febrero de 2006. En la sesión, el senador victoriense ${ }^{23}$ hizo uso de la palabra expresando que la decisión del ejecutivo le había causado, como a otros legisladores, "sorpresa" debido a que, a pesar de que consideraba que algunos artículos podrían mejorarse, "se había trabajado mucho, se habían hecho consultas a especialistas en el tema, se había analizado con todos los

22 El Proyecto, en implementación desde 2003, es ejecutado por la Organización de Estados Americanos (OEA) y es financiado por ese y otros organismos internacionales. Su objetivo es generar e implementar un modelo técnico, legal e institucional para el gerenciamiento y preservación del Acuífero Guaraní que involucre a los cuatro países apostados sobre el acuífero: Argentina, Brasil, Paraguay y Uruguay. En Argentina el actual organismo responsable del proyecto es la Sub Secretaría de Recursos Hídricos de la Secretaría de Obras Públicas del Ministerio de Planificación Federal, Inversión Pública y Servicios.

${ }^{23}$ Fue senador provincial, por el justicialismo, en los períodos 2003-2007 y 2007-2011. Según la constitución de la provincia, cada uno de los Departamentos elije un representante para la Cámara de Senadores. En el caso de la Cámara de Diputados, los representantes son elegidos por el pueblo de la provincia, en distrito único. 
PIÑEIRO CARRERAS, Julia. Turismo y recursos termales: el caso de la instalación del parque termal de la ciudad de Victoria, Entre Ríos, Argentina. Revista Brasileira de Pesquisa em Turismo. v.5, n.3, p.383405, dez.2011.

ISSN: $1982-6125$

estamentos del Gobierno Provincial que tenían injerencia en esa ley y salió una ley que creíamos que realmente reunía todas las condiciones para legislar en materia termal" (Diario de Sesiones 23/2/2006). Antes de concluir su alocución llamó a sus pares a rechazar el veto, lo cual acabó sucediendo. Con el rechazo, la Ley quedó firme. Sólo dos de sus artículos fueron luego reformados a través de una nueva Ley Provincial modificatoria, la $\mathrm{n}^{\circ} 9714^{24}$, cuyo autor fue justamente el senador victoriense, y que fue promulgada el 12 de junio.

En agosto, y en el contexto de un acto de lanzamiento ante la comunidad de un Plan Estratégico de Urbanización para la ciudad de Victoria -creado por un arquitecto paranaense que luego sería Ministro de Planeamiento, Infraestructura y Servicios provincial ${ }^{25-}$, se hizo una presentación, a través de un audiovisual, del proyecto de desarrollo del parque termal. Así, el proyecto quedaba simbólicamente ligado a lo que se proponía como un "plan de desarrollo integral". Entretanto, las obras de excavación comenzaban. Como dije anteriormente, se trataba de obras que, para el vocero de los empresarios, implicaban una inversión "de riesgo". Era "de riesgo", fundamentalmente, porque no había garantías de que el agua termal apareciera. Victoria se encontraba, explicaba en 2006 el ingeniero encargado de la excavación, en "/a última parte donde prácticamente termina el Acuífero Guaraní" (Paralelo 32, 7/10/2006). Podía suceder, también, que se encontrara agua, pero que no gozara de propiedades lo suficientemente buenas como para justificar la creación del parque. No había garantías, pero los inversores se habían arriesgado. Celebrando lo que para el vocero del grupo era una recompensa al esfuerzo y al riesgo asumido, Paralelo 32 anunció en su edición del 23 de diciembre de 2006 "iHabemus agua termal!". En 2008, el inversor me habló de los "estudios" que habían hecho. Muchos habían sido parte de las carpetas e informes que a lo largo de los años -en los que el marco legal que regulaba la explotación termal estaba siendo redefinido- habían tenido que presentar ante

${ }^{24}$ Se modificaban el artículo $27^{\circ}$ (referido a la creación del Fondo para la Conservación del Recurso Termal, el Agua, el Suelo y el Ambiente) y el $47^{\circ}$ (sobre criterios de planificación de la actividad termal).

${ }^{25}$ Para la cuestión de la planificación urbana en Victoria véase Ferrero, 2010. 
PIÑEIRO CARRERAS, Julia. Turismo y recursos termales: el caso de la instalación del parque termal de la ciudad de Victoria, Entre Ríos, Argentina. Revista Brasileira de Pesquisa em Turismo. v.5, n.3, p.383405, dez.2011.

ISSN: $1982-6125$

las autoridades provinciales en reuniones a las que, muchas veces, los acompañaba el Intendente.

Aún en mayo de ese año, cuando la obra estuviera bien avanzada solicitarían, mediante la presentación de una carpeta, la prefactibilidad pero no ya para la "exploración", sino para la "explotación" del recurso, lo cual constituía el último paso que exigía la ya entonces promulgada Ley de Termas. La entrega de la carpeta se dio en el palacio municipal donde el Intendente recibió a miembros de la empresa y al Subsecretario de Turismo de Entre Ríos, quienes unos meses antes habían compartido un viaje a España -para interiorizarse en las prácticas de turismo termal en ese país-, auspiciado por el Consejo Federal de Inversiones (CFI) y el Consejo Federal de Turismo (CFT ${ }^{26}$. Al finalizar la reunión, según publicó el departamento de prensa municipal, el Subsecretario explicó los pasos procedimentales que seguirían y el Intendente, manifestó: "[fue] una ardua tarde de trabajo en la proyección de las futuras termas de la ciudad de Victoria, un proyecto lleno de expectativas como lo fue el Hotel y el Casino Victoria, para la construcción de una ciudad turística" (www.munivictoria.gov.ar, 28/5/2008).

Despertando el contento de los involucrados en el proyecto, el Gobernador Urribarri y su Ministro Secretario de Estado de Gobierno, Justicia y Educación, firmaron el 8 de septiembre de 2009 el decreto n³.414 que dio a Victoria del Agua S.A la "Concesión para la Explotación y Preservación del Recurso Termal" por diez años renovables por otros diez. El texto expone un resumen de las actuaciones previas y dispone las "obligaciones" que en adelante corresponden a la empresa y que, en gran medida, refieren al manejo del "recurso" de modos que impidan la "degradación" del "ambiente" así como al pago de un canon por la concesión. El decreto fue entregado por el propio Gobernador en la Casa de Gobierno, en la capital entrerriana, Paraná, ante la

\footnotetext{
${ }^{26}$ Se trató de la Misión de Promoción y Buenas Prácticas en Turismo Termal a España, llevada a cabo entre el 12 y el 22 de octubre de 2007. El Subsecretario de Turismo de Entre Ríos había sido presidente del CFT durante los años 2004 y 2005. Por Victoria, habían viajado el presidente de la empresa Victoria del Agua y el entonces director del hotel asociado al casino.
} 
presencia de diversos funcionarios de la provincia, del presidente de la empresa inversora y el Intendente de Victoria.

Sólo faltaba la "habilitación definitiva", que debía ser entregada por el E.R.R.T.E.R, tal como lo disponía el decreto firmado por el Gobernador. El presidente del organismo firmó el Convenio el 18 de septiembre de 2009 en un acto desarrollado en Victoria, en el despacho del Intendente, y en el que participaron, además de los referentes del parque acuático, representantes de los distintos prestadores turísticos, y concejales locales. Según un medio electrónico local, el Intendente hizo uso de la palabra para "felicitar" a la empresa por el emprendimiento y para decir: "Esto nos pone al inicio del proyecto Victoria del Agua, después de tanta gestión, pero sabemos que hay una ley que regula, que rige, y el trámite fue un poco más complicado de lo que han sido otras termas" (www.diariovictoria.com.ar, 18/9/2009). Por su parte, el presidente del ente regulador expuso que la empresa "había cumplido con todos los requisitos que [establecía] la ley" (www.munivictoria.gov.ar, 21/9/2009) y adelantó que (ahora sí) se había decidido no emitir más habilitaciones para el desarrollo termal (www.diariovictoria.com.ar, 18/9/2009). Fue el presidente de la empresa el último en tomar la palabra para anunciar que la primera etapa del proyecto suponía una inversión de 45 millones de pesos, agradecer la "predisposición del ente" (www.munivictoria.gov.ar, 21/9/2009) y expresar que, durante el transcurso del proceso de concreción del parque, el grupo había tenido "bastante ansiedad", una "ansiedad que a veces no compatibiliza los tiempos de la actividad privada con la pública" (www.diariovictoria.com.ar, 18/9/2009).

La empresa había obtenido la última autorización que necesitaba, pero esperaba más de la provincia. El parque requería, entre otras cosas, obras de infraestructura y así lo había expresado otro de los miembros del grupo inversor durante una reunión desarrollada a fines de octubre de 2007 en la Municipalidad en la que participó Urribarri, para ese entonces Gobernador electo. Quien hablaba en nombre de la empresa, solicitó al futuro Gobernador "una serie de obras en infraestructura; tanto en el lugar como en los accesos a 
PIÑEIRO CARRERAS, Julia. Turismo y recursos termales: el caso de la instalación del parque termal de la ciudad de Victoria, Entre Ríos, Argentina. Revista Brasileira de Pesquisa em Turismo. v.5, n.3, p.383405, dez.2011.

ISSN: $1982-6125$

la ciudad y el parque" (Paralelo 32, 20/10/2007). "Un complejo termal que necesita mucho más que agua", tituló su nota sobre la reunión el periodista del semanario. Todo eso que se necesitaba formaba parte de las demandas que los inversores hacían, y harían, al Estado.

Si había obras que, se consideraba, debían demandarse directamente al gobierno provincial, otras eran de injerencia municipal. Pero algo se presentaba como problema: el predio que ocuparían las termas no formaba parte del ejido urbano de Victoria. Su incorporación legal a la ciudad fue entonces concebida y gestionada por los diversos actores locales involucrados en la concreción del proyecto como una necesidad a ser resuelta. En primer lugar, debía lograrse la sanción de una Ley Provincial ${ }^{27}$. Por ello, el senador provincial por Victoria - quien ya había tenido un rol importante en la sanción de la Ley de Termas - presentó el proyecto para la ampliación del ejido a la Cámara alta. Luego de ser discutido por la Comisión de Asuntos Constitucionales y Acuerdos, la ley recibió la media sanción del senado en agosto de 2008 donde, antes de la votación, el senador por Victoria exhortó a sus pares a aprobar su proyecto argumentando que era necesario para fortalecer "el perfil turístico" de la ciudad. Había que asegurar la extensión de un número importante de servicios e infraestructuras urbanas en gran medida correspondiente a la jurisdicción y competencia municipal entre las que mencionó "la conectividad diaria, el alumbrado público, arbolado público, barrido y limpieza urbana, recolección de residuos, telecomunicaciones, agua potable, electricidad, desagües cloacales y pluviales, transporte público de pasajeros, seguridad urbana, [y la] señalización turística y urbana". Luego de recibir la media sanción, el proyecto pasó a la Cámara de Diputados. Allí se encontraba el diputado victoriense que, según el vocero de los inversores, se había comprometido "a sacar la sanción definitiva porque [representaba] nuevas fuentes de trabajo local" (Paralelo 32, 25/10/2008). El proyecto fue

\footnotetext{
${ }^{27}$ Victoria ya contaba con un antecedente de ampliación de su ejido. En 1994, mediante la Ley Provincial № 8855 , se incluyó la zona de islas del Departamento, de una extensión de 3.760 $\mathrm{km}^{2}$.
} 
aprobado en la Cámara de Diputados el 6 de noviembre de 2008 y, en diciembre, el poder ejecutivo promulgó la Ley $n^{\circ} 9.876$ extendiendo el ejido. En provincia, el tema ya estaba dirimido. Sólo faltaba que el Concejo Deliberante de Victoria aprobara una ordenanza municipal que recogiera las determinaciones de la nueva Ley Provincial considerando los nuevos límites legales de la ciudad, lo cual ocurrió el 5 de agosto de 2009 cuando el Concejo sancionó la Ordenanza $n^{\circ} 2.777$, promulgada luego por el ejecutivo municipal que había sido el autor del proyecto. El parque termal estaba, finalmente, dentro de la ciudad.

\section{Conclusiones}

Cuando el 21 de diciembre de 2009, se realizó en Victoria el acto de "puesta en operaciones" del parque termal, muchas de las personas a las que me referí en estas páginas tuvieron un rol protagónico. En el pórtico de ingreso al parque y ante los medios de prensa y unos doscientos "invitados especiales", el presidente de la empresa, el Intendente, el Gobernador, el Secretario de Turismo de la provincia, y el Secretario de Turismo de la Nación, hicieron juntos el tradicional 'corte de cinta'. Más tarde, todos ellos se ubicaron sobre un palco para dar sus discursos. A su lado, estaban muchos de los otros involucrados en el largo proceso de instalación del parque: el senador provincial; el director del E.R.R.T.E.R; el arquitecto que había presentado el Plan Estratégico de Urbanización de Victoria (y que era ahora el Ministro de Planeamiento, Infraestructura y Servicios de la provincia ${ }^{28}$. Todos habían

\footnotetext{
28 Justamente por el rol activo que había tenido en la legislatura provincial junto al senador, un periodista reparó en la ausencia en el acto del diputado. En una reflexión editorial, el redactor de Paralelo 32 ensayaba una interpretación de esa ausencia: "Nadie me va a cambiar mi forma de ser y lo que tengo que decir lo digo", expresó el diputado (...), enojado cuando le preguntaron por su ausencia en la inauguración del complejo termal. En realidad dice que se encontraba en Paraná por el tema Presupuesto que esa noche aprobó Diputados, pero el enojo también venía porque ni siquiera se lo nombró en el acto inaugural (...). "No me molesta en lo más mínimo que [el empresario] me nombre o no", dijo el legislador que junto al senador departamental hizo lo suyo como gestor para que se destrabaran o facilitaran muchas cuestiones vinculadas con el emprendimiento. "A mí no me importa que no me nombren, lo
} 
PIÑEIRO CARRERAS, Julia. Turismo y recursos termales: el caso de la instalación del parque termal de la ciudad de Victoria, Entre Ríos, Argentina. Revista Brasileira de Pesquisa em Turismo. v.5, n.3, p.383405, dez.2011.

ISSN: $1982-6125$

ocupado un rol más o menos fundamental en la consecución del proyecto. El empresario había sido inversor y desarrollador; el Intendente había estado muy consustanciado y gestionando; el senador había tenido un papel central en la sanción de las leyes de Termas y de ampliación del ejido urbano; el director del E.R.R.T.E.R había firmado el convenio de explotación; el Ministro de Planeamiento había incluido, antes de serlo, al parque en la presentación de su Plan Estratégico; y sería, más adelante, quien llevara desde provincia distintas obras de infraestructura; el Subsecretario de Turismo nacional había apoyado el proyecto; el Subsecretario provincial había recibido el proyecto y mantenido incesantes reuniones con el Intendente y con los empresarios, con cuyo vocero había, además, compartido el viaje organizado por el CFI y el CFT. Sobre el palco, en definitiva, estaban uno al lado del otro, muchos de aquellos que venían trazando juntos los caminos del crecimiento del turismo en la localidad.

Bianchi sostiene que la atención a los empresariados locales no puede soslayar la cuestión de cómo estos se vinculan con el poder político, en la medida en que "actores locales poderosos y coaliciones empresariales pueden ser capaces de ejercer influencia sobre decisiones de planificación e inversión" (2003, p.24). Es desde ese mismo convencimiento que en este artículo he intentado mostrar en un caso concreto la centralidad que en la creación de emprendimientos turísticos tienen las relaciones entabladas por diferentes actores locales y extralocales que se conciben a sí mismos como formando parte de ámbitos distintos, el de lo privado y el de lo estatal. Se trata de relaciones que se construyen a lo largo de los años; que implican alianzas y cooperaciones; que, en muchos casos, no sólo son de carácter institucional, sino también interpersonal; y que involucran una serie de compromisos constantemente reactualizados.

que hay que hacer es seguir apoyando todo lo bueno que se haga más allá de quién lo realice" (Paralelo 32, 26/12/2009). 
PIÑEIRO CARRERAS, Julia. Turismo y recursos termales: el caso de la instalación del parque termal de la ciudad de Victoria, Entre Ríos, Argentina. Revista Brasileira de Pesquisa em Turismo. v.5, n.3, p.383405, dez.2011.

ISSN: $1982-6125$

\section{Referencias}

BARRETTO, Margarita. Turismo y Cultura. Relaciones, contradicciones y expectativas. Tenerife: PASOS Revista de Turismo y Patrimonio Cultural, 2007. Disponible en: http://www.pasosonline.org/e-books. Acceso: octubre, 2010. BIANCHI, Raoul V. Place and Power in Tourism Development. PASOS Revista de Turismo y Patrimonio Cultural. v. 1, n.1, p. 13-32, 2003. Disponible en: http://www.pasosonline.org/Publicados/1103/PS020103.pdf. Acceso: octubre, 2010.

CATULLO, M. Rosa, FERRARI; Lucrecia; MARTÍNEZ, Lucas. Proyectos de desarrollo, identidad y turismo termal. Ciudad Nueva Federación, Entre Ríos, Argentina. En: REIS, M.J.; RADOVICH, J.C.; BALAZOTE, A. (Eds.). Disputas territoriales y conflictos interétnicos en Brasil y Argentina. Córdoba: Ferreyra, 2005.

FERRERO, Laura. Disputas en torno del desarrollo y la planificación urbana en la ciudad de Victoria, Entre Ríos: el papel de los funcionarios y expertos en la producción de consenso. En: VI JORNADAS DE INVESTIGACIÓN EN ANTROPOLOGÍA SOCIAL, SEANSO, ICA, FFyL, UBA, 2010, Buenos Aires. Actas... Buenos Aires: FFyL. 1 CD-ROM.

GAZTAÑAGA, Julieta. El trabajo político y sus obras. Una etnografía de tres procesos políticos en a Argentina contemporánea. Buenos Aires: GiaperAntropofagia, 2010.

INDEC. Censo Nacional de Población, Hogares y Viviendas de 2001. Disponible en http://www.indec.gov.ar/. Acceso: noviembre, 2011.

NOGUÉS PEDREGAL, Antonio M. Una genealogía de la difícil relación entre antropología social y turismo. PASOS Revista de Turismo y Patrimonio Cultural, v.7, n.1, p. 43-56, 2009.

PIÑEIRO CARRERAS, Julia. Una ciudad 'condenada al éxito': crecimiento turístico y transformaciones sociales. En: VIII REUNIÓN DE ANTROPOLOGÍA DEL MERCOSUR, UNSAM, Buenos Aires, 2009. Actas... San Martín: UNSAM. 1 CD-ROM.

PIÑEIRO CARRERAS, Julia. Los emprendimientos turísticos y sus efectos: el caso del Hotel-Casino en Victoria, Entre Ríos, Argentina. Estudios y Perspectivas en Turismo, v. 20, n. 5, p. 1069-1083, 2011. Disponible en: http://www.estudiosenturismo.com.ar/PDF/V20/N05/v20n5a06.pdf.

PIÑEIRO CARRERAS, Julia. Una ciudad condenada al éxito: etnografía del proceso de construcción social de Victoria como destino turístico. Tesis de Maestría IDES-IDAES/UNSAM. Dir: Dra. Ana Rosato, 2011. Inédito.

RAMÍREZ, Lucas. Turismo, desarrollo y transformación territorial. Federación (provincia de Entre Ríos): de la ciudad relocalizada a la "ciudad termal". En: CONGRESO DE GEOGRAFÍA DE LAS UNIVERSIDADES PÚBLICAS, Universidad Nacional del Litoral, Santa Fe, 2011.

WALLINGRE, Noemí. Evolución del desarrollo del turismo en la provincia de Entre Ríos. Revista Tiempo de Gestión, Facultad de Ciencias de la Gestión. Universidad Autónoma de Entre Ríos, n.5., 2008 
PIÑEIRO CARRERAS, Julia. Turismo y recursos termales: el caso de la instalación del parque termal de la ciudad de Victoria, Entre Ríos, Argentina. Revista Brasileira de Pesquisa em Turismo. v.5, n.3, p.383405, dez.2011.

ISSN: $1982-6125$

WALLINGRE, Noemí. Indagaciones sobre los cambios acontecidos en el desarrollo del turismo en la ciudad de San Jose, provincia de Entre Rios, Argentina. Revista Brasileira de Pesquisa em Turismo, v.5, n.2, p.271- 296, 2011.

Disponible

en: http://www.revistas.univerciencia.org/turismo/index.php/rbtu/article/view/412 /472. Acceso en: octubre, 2011.

\section{Fuentes}

"iHabemus agua termal!", Paralelo 32, Edición Victoria-Nogoyá, 23 de diciembre de 2006.

"Aguas Termales y Hoteles. Se caen y se levantan propuestas todas las semanas", Paralelo 32, Edición Victoria-Nogoyá, 23 de octubre de 2004.

"Aguas termales. Replantean la explotación del recurso", Paralelo 32, Edición Victoria-Nogoyá. 22 de mayo de 2004.

"Caleidoscopio", Paralelo 32, Edición Victoria-Nogoyá, 26 de diciembre de 2009.

"El gruyere termal", Paralelo 32. Edición Victoria-Nogoyá, 31 de enero de 2004.

"El proyecto termal ya fue presentado a la provincia", Paralelo 32. Edición Victoria-Nogoyá, 22 de mayo de 2004.

"El termalismo entrerriano en la encrucijada", Paralelo 32, Edición VictoriaNogoyá, 25 de septiembre de 2004.

"En menos de dos meses podrían llegar al acuífero", Paralelo 32, Edición Victoria-Nogoyá, 7 de octubre de 2006.

"Firma del convenio de explotación de Victoria del Agua", Archivo de Noticias de la Municipalidad de Victoria, 21 de septiembre de 2009. Disponible en: http://www.munivictoria.gov.ar/municipalidad/archivosanteriores/Septiembre2 009/. Acceso: 24 de septiembre de 2009.

"Parque recreativo temático termal en victoria", Archivo de Noticias de la Municipalidad de Victoria, 28 de mayo de 2008. Disponible en: http://www.munivictoria.gov.ar/municipalidad/archivosanteriores/mayo2008/. Acceso: 2 de junio de 2008.

"Stur confesó que lo tomó por sorpresa el veto total a la Ley de Termas", Paralelo 32, Edición Victoria-Nogoyá, 4 de febrero de 2006.

"Termas", Paralelo 32, Edición Victoria-Nogoyá. Sección Grageas, 24 agosto de 2004.

"Un complejo termal que necesita mucho más que agua", Paralelo 32. Edición Victoria-Nogoyá, 20 de octubre de 2007.

"Un proyecto termal en marcha", Paralelo 32. Edición Victoria-Nogoyá, 24 de abril de 2004.

"Veto termas. En Se realizó una nueva licitación participando dos oferentes", Paralelo 32, Edición Victoria-Nogoyá, 28 de enero de 2006.

"Victoria del Agua podrá explotar turísticamente las termas", www.diariovictoria.com, 18 de septiembre de 2009. Disponible en: 
PIÑEIRO CARRERAS, Julia. Turismo y recursos termales: el caso de la instalación del parque termal de la ciudad de Victoria, Entre Ríos, Argentina. Revista Brasileira de Pesquisa em Turismo. v.5, n.3, p.383405, dez.2011.

ISSN: $1982-6125$

http://www.diariovictoria.com.ar/2009/09/victoria-del-agua-podra-explotarturisticamente-las-termas/. Acceso: 20 de septiembre de 2009.

"Victoria recibirá 2009 con la primera etapa del complejo termal habilitado", Paralelo 32, Edición Victoria-Nogoyá, 25 de octubre de 2008.

ARGENTINA. Constitución Nacional. Argentina, Segunda Parte: Autoridades de la Nación. Titulo Segundo. Gobiernos de provincia Art. $124^{\circ}$. Disponible en:

ENTRE RÍOS (Provincia). Asamblea Legislativa. Diario de Sesión $126^{\circ}$ período legislativo; reunión Nro. 16, $2^{\text {da }}$ sesión. 23 de febrero de 2006. Disponible en: http://www.senadoer.gov.ar/galeria/sesion/126-16.pdf. Acceso: julio 2011.

ENTRE RÍOS (Provincia). Cámara de Diputados. Diario de Sesión 1290 período legislativo; reunión Nro. 08, $7^{\text {ma }}$ Sesión Ordinaria. 6 de noviembre de 2008. Disponible en: http://www.hder.gov.ar/diarios_sesiones.php. Acceso: julio, 2011.

ENTRE RÍOS (Provincia). Decretos Nros. 7137/04; 1355/05; 37/2006; 3414/09. Disponibles en: http://www.entrerios.gov.ar/wsdecreto. Acceso: mayo, 2011.

ENTRE RÍOS (Provincia). Ley No 9678, del 27 de febrero de 2006. Disponible en: http://www.senadoer.gov.ar/galeria/ley/1242047588.pdf. Acceso: 2 de mayo de 2011.

ENTRE RÍOS (Provincia). Ley N9714, del 12 de junio de 2006. Disponible en: http://www.senadoer.gov.ar/galeria/ley/1242055708.pdf. Acceso: 2 de mayo de 2011.

ENTRE RÍOS (Provincia). Ley N9876, del 10 de diciembre de 2008. Disponible en: http://www.senadoer.gov.ar/galeria/sesion/126-16.pdf. Acceso: 2 de mayo de 2011.

http://www.senado.gov.ar/web/interes/constitucion/gobiernos.php. Acceso: julio de 2011.

VICTORIA (Municipio), ENTRE RÍOS. Concejo Deliberante. Ordenanza Nro. 2.472/05, del 6 de febrero de 2005. Disponible en: http://www.munivictoria.gov.ar/ordmunicipal2472.pdf. Acceso: octubre, 2010. VICTORIA (Municipio), ENTRE RÍOS. Concejo Deliberante. Ordenanza Nro. $2.777 / 09$.

Artigo recebido em novembro de 2011.

Aprovado para publicação em dezembro de 2011. 